\title{
Impact force reduction strategies to achieve safer human-robot collisions
}

\author{
Behrad Rouzbeh and Gary M. Bone* \\ Department of Mechanical Engineering \\ McMaster University \\ Hamilton, Canada \\ *Corresponding author. E-mail: gary@mcmaster.ca
}

\begin{abstract}
The increasing use of robots operating close to people has made human-robot collisions more likely. In this paper, strategies intended to reduce the impact force to a safe level, without sacrificing the robot's performance, are investigated. The strategies can be applied to a robot arm without modifying its internal hardware. They include the existing strategies: lowering the actuator controller's stiffness; actuator switched off upon impact detection; withdrawing the arm upon impact detection; and adding a compliant cover. We also propose the novel strategy of limiting the controller's feedback term. The collision scenario studied is a robot arm colliding with a person's constrained head. An improved lumped parameter model of the constrained impact is proposed. Simulation results are included for a UR5 collaborative robot. Sixteen combinations of the impact force reduction strategies are compared. The results show that using a high stiffness controller with a feedback limit and compliant cover reduces the impact force to a safe level, and achieves precise trajectory tracking.
\end{abstract}

Keywords-collaborative robot; compliant covering; humanrobot collision; impact force; robot control; robot safety

\section{INTRODUCTION}

In emerging applications, such as collaborative robots and service robots, robot arms (also termed "manipulators") may operate in close proximity with people. This proximity makes human-robot collisions much more likely so the ability to reduce the human-robot impact force to a safe level is a critical requirement for these robots. Research on the design and control of safe collaborative robots (also termed "humanfriendly robots" in the literature) aims to address this safety requirement without sacrificing the robot's performance and functionality.

Previous researchers have proposed a wide variety of approaches for reducing the human-robot impact force. In [1]

Research sponsored by the Natural Sciences and Engineering Research Council of Canada (NSERC) through a Discovery Grant. the impact force is reduced by applying a time-varying limit to the actuator torques based on a dynamic model of the robot. While this can work in certain situations, an undesirable reduction in the precision of the robot's trajectory tracking may also occur when the actuator torques are limited. Reducing the inertia of the robot is very effective at reducing the impact force. The robot developed by DLR [2] is a prime example of this approach whose special design and use of lightweight materials, such as carbon fiber, produced a very low inertia arm. However, this is a costly solution and obviously cannot be used to improve the safety of conventional robots. Reducing the stiffness of the transmission between the actuators and links is another approach for improving the collision safety, e.g. [3][4]. With conventional robots the transmission between each actuator and link is very stiff so both of their inertias contribute to the severity of the collision. The objective of variablestiffness actuation (VSA) is to dynamically decouple the actuator's rotor inertia from the link's inertia. Since the contact occurs between the human and the link, and the link's inertia is lower than the combined inertia, the impact force will be reduced by the VSA's decoupling effect. The lower the stiffness the lower the force, however low transmission stiffness also tends to lower the precision of the robot's position control. Similarly, a lower arm velocity also reduces the impact force, but clearly sacrifices the robot's performance. The VSA mitigates these two problems by lowering the stiffness when the velocity is high, and increasing the stiffness when the velocity is low. This addresses the safety aspect, but will also cause the tracking precision to deteriorate during the higher velocity sections of its motion trajectories. Furthermore, using VSAs for the robot's six joints will increase the complexity, cost and inertia of the arm. Adding an electromechanical clutch between the link and actuator is an alternate approach to decouple the actuator's and link's inertias [5][6]. This behaves like a VSA with two stiffnesses and is termed a "series clutch actuator" (SCA). When clutch is engaged the transmission's stiffness is very high, and when it is disengaged the stiffness drops to zero. Unlike a VSA, the SCA should not affect the robot's performance during normal conditions since the clutch should only disengage when a collision happens. The clutch's torque threshold can also be 
varied to satisfy both the safety and performance requirements [5]. Unfortunately, using SCAs will increase the complexity, cost and inertia of the robot arm.

While the approaches in [2]-[6] all have merit, we are interested in methods for reducing the impact force that can be applied to existing robot arms with little or no modifications to the arm's hardware. The actuator torque limiting method in [1] is such an approach, but has serious side effects as previously discussed. A better approach is to add a collision detection and reaction strategy to the robot's control software, e.g. [7]-[9]. The detection can use either the robot's existing sensors [7][8] or externally mounted sensors [9]. The former approach requires a dynamic model of the robot while the latter does not. Using either approach detection times of a few ms can be achieved [7]-[9]. Various reaction strategies may be executed after contact detection to reduce the impact force, such as: braking to a stop [7][9], switching the actuator off [8], or having the arm reflexively withdrawn from the location of the impact [8]. Another method for increasing the safety of an existing robot is to add a compliant cover over its surface, e.g. [7][10][11]. The cover's stiffness can be chosen to satisfy a safety constraint without being excessively thick [11]. The compliant cover cannot be used with the end effector if it interferes with its function. If this is the case, quick release mechanisms, such as the QuickSTOP collision device (Applied Robotics Inc.), can be used to increase the safety of collisions with the end effector.

In this paper we will investigate strategies intended to reduce the impact force when a robot collides with a human's head, while maintaining the robot's performance during normal conditions. These can be applied to an existing robot without modifying its internal hardware. They include the previously applied strategies: lowering the actuator controller's stiffness, actuator switched off by controller upon impact detection, withdrawing the arm as fast as possible upon impact detection, and adding a compliant cover. We also propose the novel strategy of limiting the magnitude of the feedback term used with the controller.

The collision scenario is described in section II. In section III the impact model for this scenario is derived. Section IV covers the controller design. Simulation results are presented and discussed in section $\mathrm{V}$ and conclusions are drawn in section VI.

\section{COLLISION SCENARIO}

Although the human-robot impact could occur almost anywhere on the human's body we will investigate impact with the human head since it is the most likely to result in serious injuries. The collision scenarios being studied are illustrated in Fig. 1. We assume that the impact takes place at the tip of the forearm link when the robot arm is fully extended as shown. This contact location and elbow angle maximizes the moment of inertia of the arm which will produce the maximum impact force, all other parameters being equal. We also assume the impact direction and human's surroundings do not allow the head to move - this is termed a "constrained impact" and produces much larger forces than the unconstrained case where the head is allowed to move [11].

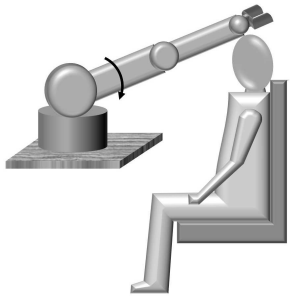

(a)

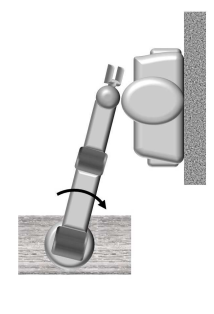

(b)
Figure 1. Illustration of the collision scenarios being studied. a) Head impact caused by robot's shoulder joint movement. b) Head impact caused by robot's waist joint movement.

The robot is assumed to be a standard articulated arm with six or seven motorized rotary joints. The impact may be caused by either the rotation of the robot's shoulder joint (Fig. 1a) or its waste joint (Fig. 1b). The elbow joint is assumed to be infinitely stiff which is a conservative assumption.

\section{HUMAN-ROBOT IMPACT MODELING}

As is common practice in the literature [3][4][10][11][12], the human-robot impact dynamics in the normal direction are modelled by a linear one dimensional lumped parameter model consisting mainly of masses and springs. Fig. 2 shows the schematic for the linear robot-head impact model. The actuator force (commanded by the controller), equivalent masses of the actuator and link are included. The mass of the head is shown but not included in our equations since it is constrained. Knowing the elastic modulus and contact area of the compliant material, the compliant cover can be modeled as a spring acting between the link and head. The human head's stiffness is also modelled as a spring. Its value for robot-head contact is included in the international technical specification for collaborative robots [12]. Including this spring makes the model more realistic than previous models that assumed the skull is perfectly rigid. Displacement due to transmission deflection adds one degree of freedom to the model which is shown as spring acting between the actuator position and the robotic arm position. Most existing robots use a low-backlash high-precision transmission at each joint which has a high stiffness compared to the controller's stiffness (due to feedback). Thus the transmission spring can be reasonably neglected, making the actuator and links act as a single mass. Fig. 3 shows the simplified model that is employed in the remainder of this paper. is:

The conventional position controller equation for the joint

$$
\tau_{\text {cont }}=K_{p}\left(\theta_{d}-\theta_{r}\right)+K_{d}\left(\dot{\theta}_{d}-\dot{\theta}_{r}\right)+I_{r} \ddot{\theta}_{d}
$$

subject to:

$$
\left|\tau_{\text {cont }}\right| \leq \tau_{\max }
$$

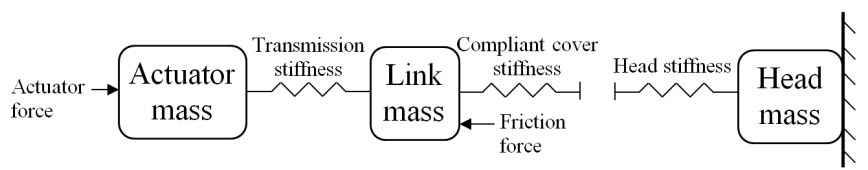

Figure 2. Linear robot link-head blunt constrained impact model. 


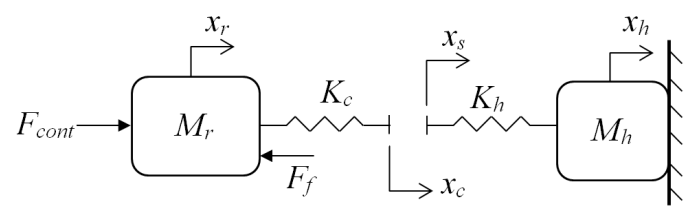

Figure 3. Simplified model for robot link-head blunt constrained impact.

where $\tau_{\text {cont }}$ is the joint torque to be applied by the actuator; $\tau_{\max }$ is the maximum actuator torque; $\theta_{r}$ is the joint angle; $\theta_{d}$ is the desired angle; $K_{p}$ and $K_{d}$ are the proportional and derivative feedback gains respectively; $I_{r}$ is the combined inertias of the actuator and links; and $I_{r} \ddot{\theta}_{d}$ is the feedforward term used to compensate the inertial torque. Assuming the post-impact deflections are small, the equivalent controller force in the normal direction at the point of contact is:

$$
F_{\text {cont }}=K_{\text {cont }}\left(x_{d}-x_{r}\right)+C_{\text {cont }}\left(\dot{x}_{d}-\dot{x}_{r}\right)+M_{r} \ddot{x}_{d}
$$

where $K_{\text {cont }}=K_{p} / L^{2}, C_{\text {cont }}=K_{d} / L^{2}, M_{r}=I_{r} / L^{2}, x_{d}=\theta_{d} L$, $x_{r}=\theta_{r} L$ and $L$ is the distance from the impact point to the joint. If all displacements are relative to $x_{h}$, the robot's equation of motion is:

$$
M_{r} \ddot{x}_{r}=F_{c o n t}-F_{f}-F_{i}
$$

where $F_{f}$ is the friction force and $F_{i}$ is the impact force. The impact force is given by:

$$
F_{i}=\left\{\begin{array}{cc}
K_{h c} x_{r} & x_{r}>0 \\
0 & x_{r} \leq 0
\end{array}\right.
$$

where:

$$
K_{h c}=K_{h} K_{c} /\left(K_{h}+K_{c}\right)
$$

Substituting (3) and (5) into (4) and rearranging gives:

$$
M_{r} \ddot{x}_{r}+C_{r} \dot{x}_{r}+K_{r} x_{r}=M_{r} \ddot{x}_{d}+C_{\text {cont }} \dot{x}_{d}+K_{c o n t} x_{d}-F_{f}
$$

where:

$$
\begin{gathered}
C_{r}=C_{c o n t} \\
K_{r}=\left\{\begin{array}{ll}
K_{h c}+K_{c o n t} & x_{r}>0 \\
K_{c o n t} & x_{r} \leq 0
\end{array}\right. \text { and }
\end{gathered}
$$

The friction force is modelled as the sum of dry and viscous components as follows:

$$
F_{f}=\left\{\begin{array}{c}
f_{k} \operatorname{sign}\left(\dot{x}_{r}\right)+k_{v} \dot{x}_{r} \quad \dot{x}_{r} \neq 0 \\
F_{\text {cont }}-F_{i} \quad \dot{x}_{r}=0 \wedge\left|F_{\text {cont }}-F_{i}\right|<f_{s} \\
f_{s} \operatorname{sign}\left(F_{\text {cont }}-F_{i}\right) \quad \text { otherwise. }
\end{array}\right.
$$

where $f_{k}$ is the kinetic friction force, $f_{s}$ is the static friction force and $k_{v}$ is the coefficient of viscous friction.

Lastly, the deflections of the compliant cover and head may be calculated using (11) and (12), respectively.

$$
\begin{gathered}
\Delta x_{c}=x_{c}-x_{r}=F_{i} / K_{c} \text { and } \\
x_{s}=F_{i} / K_{h}
\end{gathered}
$$

\section{CONTROLLER DESIGN}

The position controller can be designed for trajectory tracking by specifying the desired closed-loop bandwidth, $f_{b w}$, and damping ratio, $\zeta$. The corresponding controller gains are given by:

$$
\begin{gathered}
K_{p}=I_{r} \omega_{n}{ }^{2} \\
K_{d}=2 \zeta \sqrt{K_{p} I_{r}}
\end{gathered}
$$

where:

$$
\omega_{n}=2 \pi f_{b w} /\left(\zeta+\sqrt{1+\zeta^{2}}\right)
$$

The proposed feedback limited (FBL) controller is obtained by replacing (1) with:

$$
\begin{gathered}
\tau_{\text {cont }}=\tau_{f b}+\tau_{f f} \\
\tau_{f b}=K_{p}\left(\theta_{d}-\theta_{r}\right)+K_{d}\left(\dot{\theta}_{d}-\dot{\theta}_{r}\right) \text { and } \\
\tau_{f f}=I_{r} \ddot{\theta}_{d}
\end{gathered}
$$

subject to (2) and:

$$
\left|\tau_{f b}\right| \leq \tau_{\text {lim }}
$$

where $\tau_{\text {lim }}$ is the feedback torque limit.

\section{Simulations}

Simulations were created to compare the position control performance and impact forces produced under a variety of conditions. The simulated robot is a Universal Robot model UR5 which is one of the most common collaborative robots in use today. The robot's commanded trajectory is defined such that it collides with the head at a velocity of $0.25 \mathrm{~m} / \mathrm{s}$ which is the highest speed permitted by the Canadian [13] and international safety standards [14] when a human is within reach of a robot arm. Two different sets of controller gains $K_{p}$ and $K_{d}$ were used, one for a high bandwidth, high stiffness controller (HSC) and the other for a lower stiffness, lower bandwidth controller (LSC). The two bandwidths for the HSC and LSC are $50 \mathrm{~Hz}$ and $5 \mathrm{~Hz}$, respectively. The corresponding stiffnesses are $355 \mathrm{kN} / \mathrm{m}$ and $3.55 \mathrm{kN} / \mathrm{m}$, respectively. The other simulation parameters are listed in Table I.

Several impact conditions have been simulated and comparisons made. The HSC and HSC were simulated with and without compliant cover (CC). The other impact force reduction strategies simulated are: limiting the controller feedback force applied to the link (FBL); switching off the actuator when the impact is detected (SOA); and implementing an arm withdrawal reflex (AWR) by applying the maximum actuator torque in the opposite direction. The impact detection 
TABLE I. SIMULATION PARAMETERS

\begin{tabular}{|c|c|c|}
\hline Parameter & Value & Description \\
\hline$d t$ & $0.01 \mathrm{~ms}$ & Integration timestep \\
\hline$f_{k}$ & $19.4 \mathrm{~N}$ & Kinetic friction force ${ }^{a}$ \\
\hline$f_{s}$ & $19.4 \mathrm{~N}$ & Static friction force ${ }^{\mathrm{a}}$ \\
\hline$K_{c}$ & $25 \mathrm{kN} / \mathrm{m}$ & Compliant cover stiffness [11] \\
\hline$K_{h}$ & $150 \mathrm{kN} / \mathrm{m}$ & Head stiffness [12] \\
\hline$k_{v}$ & $0.554 \mathrm{Ns} / \mathrm{m}$ & Coefficient of viscous friction ${ }^{b}$ \\
\hline$L$ & $0.85 \mathrm{~m}$ & $\begin{array}{l}\text { Distance from joint to impact } \\
\text { location on the robot [16 }\end{array}$ \\
\hline$M_{r}$ & $9.57 \mathrm{~kg}$ & Equivalent robot mass ${ }^{\mathrm{c}}$ \\
\hline$T_{d}$ & $5 \mathrm{~ms}$ & Impact detection delay \\
\hline$T_{s}$ & $1 \mathrm{~ms}$ & Controller sampling period \\
\hline$\zeta$ & 0.7 & Controller damping ratio \\
\hline$\tau_{\text {lim }}$ & $20 \mathrm{Nm}$ & Controller feedback limit \\
\hline$\tau_{\max }$ & $150 \mathrm{Nm}$ & Maximum joint torque [15] \\
\hline
\end{tabular}

delay for the AWR was assumed to be $5 \mathrm{~ms}$. The robot was simulated tracking a trapezoidal velocity trajectory before collision and the tracking root-mean-squared-error (RMSE) values were computed. To make the tracking more realistic the friction coefficients were subjected to a $5 \%$ random variation from the nominal. Table II lists the results for the cases simulated.

The International Organization for Standardization (ISO) specifies $130 \mathrm{~N}$ as the maximum permissible force for the skull and forehead. Studying the results in the table, it can be observed that the robot with HSC and no CC, FBL or AWR exerted an impact force of $410 \mathrm{~N}$ (case 1), well beyond the ISO safety limit. This $410 \mathrm{~N}$ value is used as the benchmark for comparison. Using the LSC reduced the impact force $24 \%$ to $313 \mathrm{~N}$ (case 9), while adding a carefully selected limit with the
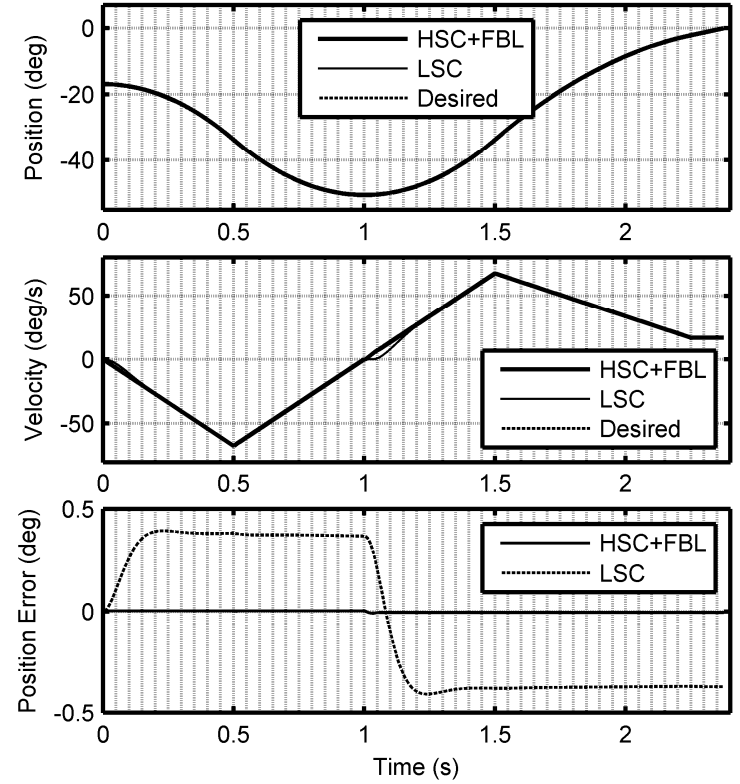

Figure 4. Comparison of the joint's motion tracking performances before impact for higher stiffness controller with feedback limit (HSC+FBL) and the lower stiffness controller (LSC).
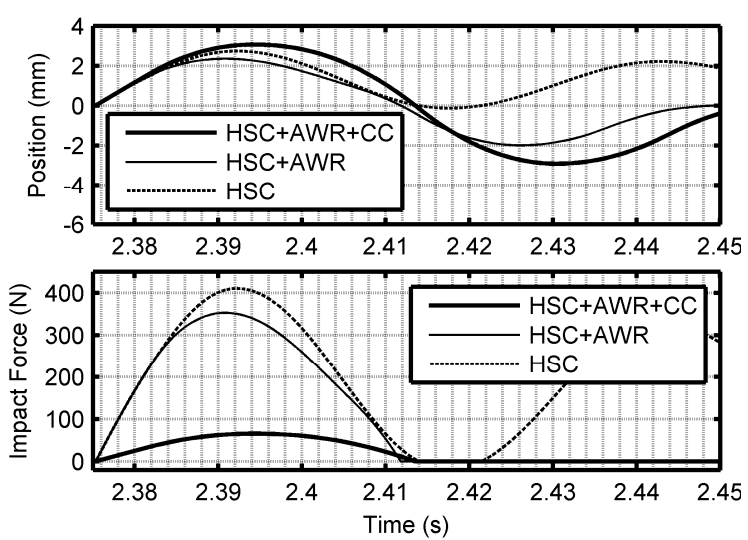

Figure 5. Post-impact link tip position and impact force for the high stiffness controller with different features: with no impact reduction feature (HSC), with arm withdrawal reflex (HSC+AWR), and with arm withdrawal reflex and compliant cover (HSC+AWR+CC).

FBL strategy reduced the force $26 \%$ to $303 \mathrm{~N}$ (case 2). Although these two strategies have a similar effect on the impact force, the RMSE before the impact is $99 \%$ smaller when FBL is used (case 2 vs. case 9). Fig. 4 compares the tracking performance for these two cases. The larger position and velocity errors with LSC are obvious in this figure. Adding $\mathrm{CC}$ to the robot with HSC reduced the impact force $36 \%$ (case 5) and when combined with FBL, the impact force reduction is $72 \%$ (case 6) and the force of $117 \mathrm{~N}$ is less than the ISO limit.

For the uncovered robot with HSC, SOA (case 3) and AWR (case 4) produced similar forces to FBL (case 2) since the impact force rises so fast that it is near its peak before the impact is detected by the SOA and AWR strategies. However when $\mathrm{CC}$ is used with HSC the force rises more slowly, allowing SOA (case 7) and AWR (case 8) to reduce the impact force by $75 \%$ and $84 \%$, respectively, to values well within the ISO safety limit. Fig. 5 compares the effectiveness of AWR strategy with and without $\mathrm{CC}$ to the benchmark case (without any force reduction method) for the HSC. With the benchmark case, in addition to large forces it can be observed that repeated impacts occur.

Regarding the LSC, cases 9-16 show a similar pattern to the corresponding HSC cases 1-8. Fig. 6 shows the results for the
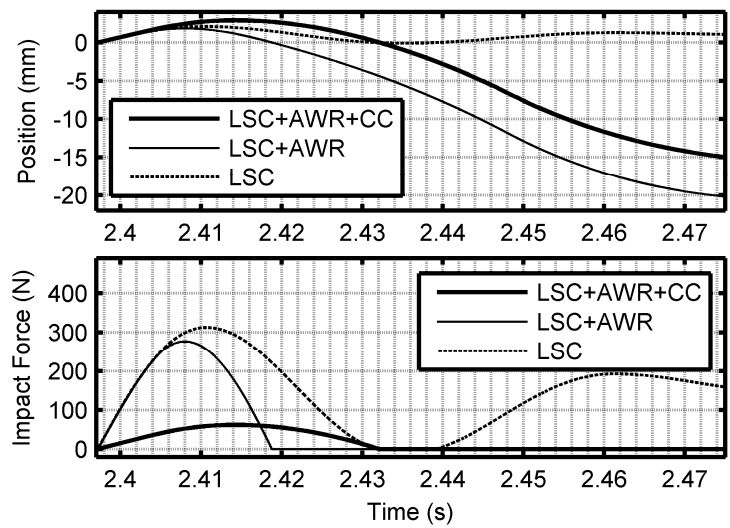

Figure 6. Post-impact link tip position and impact force for the low stiffness controller with different features: with no impact reduction feature (LSC), with arm withdrawal reflex (LSC+AWR), and with arm withdrawal reflex and compliant cover ( $\mathrm{LSC}+\mathrm{AWR}+\mathrm{CC})$. 
LSC and the same combinations of AWR and CC as in Fig. 5. Compared with Fig. 5, the same improvement can be observed when CC is added, with the main difference being the arm is withdrawn much further back after the impact when using the LSC. From these figures and the tabulated results it can be observed that the LSC provides no significant safety advantage when CC and AWR are used together.

\section{CONCLUSION}

Several strategies for reducing the impact force caused by the collision of a robot arm with a constrained human head were investigated. The effect of the strategies on the robot's trajectory tracking precision during normal operation was also studied. An improved lumped parameter model for the constrained impact was proposed for simulating the collisions. Sixteen combinations of impact reduction strategies were simulated and compared for a UR5 collaborative robot. Compared to the uncovered robot with the HSC, the LSC (with $1 \%$ of the HSC's stiffness) only made the impact force slightly smaller, and made the tracking errors much larger. The SOA and AWR strategies were effective when CC was used, but have the disadvantage of requiring rapid impact detection. Combining the proposed FBL strategy with HSC and $\mathrm{CC}$ reduced the impact force to less than the ISO safety limit and achieved precise trajectory tracking. This combination also does not require any impact detection.

\section{REFERENCES}

[1] J. Heinzmann and A. Zelinsky, "Quantitative safety guarantees for physical human-robot interaction," Int. J. Rob. Res., vol. 22, no. 7-8, pp. 479-504, 2003.

[2] G. Hirzinger, A. Albu-Schäffer, M. Hähnle, I. Schaefer, and N. Sporer, "On a new generation of torque controlled light-weight robots," in Proc. IEEE Int. Conf. Robot. Automat., 2001, pp. 3356-3363.

[3] A. Bicchi and G. Tonietti, "Fast and 'Soft-Arm' Tactics," IEEE Robot. Autom. Mag., vol. 11, no. 2, pp. 22-33, Jun. 2004.
[4] D. Hyun, H. S. Yang, J. Park, and Y. Shim, "Variable stiffness mechanism for human-friendly robots," Mech. Mach. Theory, vol. 45, no. 6, pp. 880-897, 2010.

[5] N. Lauzier and C. Gosselin, "Performance indices for collaborative serial robots with optimally adjusted series clutch actuators," J. Mech. Robot., vol. 4, no. 2, p. 21002, 2012.

[6] M. Moghani and M. R. Kermani, "Design and development of a hybrid magneto-rheological clutch for safe robotic applications," in Proc. IEEE Int. Conf. Robot. Automat., 2016, pp. 3083-3088.

[7] K. Suita, Y. Yamada, N. Tsuchida, K. Imai, H. Ikeda, and N. Sugimoto, "A failure-to-safety' Kyozon' system with simple contact detection and stop capabilities for safe human-autonomous robot coexistence," in Proc. IEEE Int. Conf. Robot. Automat., 1995, vol. 3, pp. 3089-3096.

[8] A. De Luca, A. Albu-Schaffer, S. Haddadin, and G. Hirzinger, "Collision detection and safe reaction with the DLR-III lightweight manipulator arm," in Proc. IEEE/RSJ Int. Conf. on Intell. Robots and Syst., 2006, pp. 1623-1630.

[9] S. Jeong and T. Takahashi, "Impact force reduction of manipulators using a dynamic acceleration polytope and flexible collision detection sensor," Adv. Robot., vol. 23, no. 3, pp. 367-383, 2009.

[10] S. Haddadin, T. Laue, U. Frese, S. Wolf, A. Albu-Schäffer, and G. Hirzinger, "Kick it like a safe robot: Requirements for 2050," Robot. Auton. Syst., vol. 57, pp. 761-775, 2009.

[11] L. Zeng and G. M. Bone, "Design of elastomeric foam-covered robotic manipulators to enhance human safety," Mech. Mach. Theory, vol. 60, pp. 1-27, 2013.

[12] ISO/TS 15066:2016, Robots and robotic devices - Collaborative robots. International organization for standardization, 2016.

[13] CAN/CSA-Z434-14 - Industrial robots and robot systems. Canadian Standards Association, 2014.

[14] ISO 10218-1:2011, Robots for industrial environments - Safety requirements - Part I: Robot. International organization for standardization, 2011.

[15] K. Kufieta, "Force Estimation in Robotic Manipulators: Modeling, Simulation and Experiments, UR5 as a case study," Diploma Thesis, Norwegian University of Science and Technology, Department of Engineering Cybernetics, Norway, Jan. 29, 2014.

[16] P. M. Kebria, S. Al-wais, H. Abdi and S. Nahavandi, "Kinematic and dynamic modelling of UR5 manipulator," in Proc. IEEE Int. Conf. Syst., Man, and Cyber., 2016, pp. 4239-4234.

TABLE II. SIMULATION RESULTS

\begin{tabular}{|c|c|c|c|c|c|c|c|c|}
\hline $\begin{array}{l}\text { Case } \\
\text { Number }\end{array}$ & $\begin{array}{c}\text { Controller Stiffness, } \\
K_{\text {cont }}(\mathrm{kN} / \mathrm{m})\end{array}$ & $\begin{array}{c}\text { Controller } \\
\text { Bandwidth }(\mathrm{Hz})\end{array}$ & $\begin{array}{l}\text { Switch Off } \\
\text { Actuator }\end{array}$ & $\begin{array}{c}\text { Arm } \\
\text { Drawback }\end{array}$ & $\begin{array}{c}\text { Compliant } \\
\text { Cover }^{\mathrm{a}}\end{array}$ & $\begin{array}{c}\text { Limit on } \\
\text { Feedback }\end{array}$ & $\begin{array}{c}\text { Tracking } \\
\text { RMSE (deg) }\end{array}$ & $\begin{array}{c}\text { Peak Impac } \\
\text { Force }(\mathrm{N})\end{array}$ \\
\hline 1 & 355 & 50 & No & No & No & No & $3.73 \mathrm{E}-3$ & 410 \\
\hline 2 & 355 & 50 & No & No & No & Yes & $3.79 \mathrm{E}-3$ & 303 \\
\hline 3 & 355 & 50 & Yes & No & No & No & $3.73 \mathrm{E}-3$ & 329 \\
\hline 4 & 355 & 50 & No & Yes & No & No & $3.73 \mathrm{E}-3$ & 353 \\
\hline 5 & 355 & 50 & No & No & Yes $(10.5 \mathrm{~mm})$ & No & $3.73 \mathrm{E}-3$ & 264 \\
\hline 6 & 355 & 50 & No & No & Yes $(4.7 \mathrm{~mm})$ & Yes & $3.79 \mathrm{E}-3$ & 117 \\
\hline 7 & 355 & 50 & Yes & No & Yes $(4.1 \mathrm{~mm})$ & No & $3.73 \mathrm{E}-3$ & 103 \\
\hline 8 & 355 & 50 & No & Yes & Yes $(2.6 \mathrm{~mm})$ & No & $3.73 \mathrm{E}-3$ & 66 \\
\hline 9 & 3.55 & 5 & No & No & No & No & 0.362 & 313 \\
\hline 10 & 3.55 & 5 & No & No & No & Yes & 0.405 & 303 \\
\hline 11 & 3.55 & 5 & Yes & No & No & No & 0.362 & 299 \\
\hline 12 & 3.55 & 5 & No & Yes & No & No & 0.362 & 277 \\
\hline 13 & 3.55 & 5 & No & No & Yes $(5.3 \mathrm{~mm})$ & No & 0.362 & 132 \\
\hline 14 & 3.55 & 5 & No & No & Yes $(4.7 \mathrm{~mm})$ & Yes & 0.405 & 116 \\
\hline 15 & 3.55 & 5 & Yes & No & Yes $(4.1 \mathrm{~mm})$ & No & 0.362 & 102 \\
\hline 16 & 3.55 & 5 & No & Yes & Yes $(2.5 \mathrm{~mm})$ & No & 0.362 & 62 \\
\hline
\end{tabular}

a. Maximum compliant cover deflections during impact are shown in brackets. 